\title{
On the Fractional View Analysis of Keller-Segel Equations with Sensitivity Functions
}

\author{
Haobin Liu, ${ }^{1,2}$ Hassan Khan $\mathbb{D}^{3},{ }^{3}$ Rasool Shah $\mathbb{D}^{3}{ }^{3}$ A. A. Alderremy, ${ }^{4}$ Shaban Aly, ${ }^{5}$ \\ and Dumitru Baleanu, ${ }^{6,7}$ \\ ${ }^{1}$ Data Recovery Key Laboratory of Sichuan Province, Neijiang Normal University, Neijiang 641000, Sichuan, China \\ ${ }^{2}$ School of Mathematics and Information Sciences, Neijiang Normal University, Neijiang 641000, Sichuan, China \\ ${ }^{3}$ Department of Mathematics, Abdul Wali Khan University Mardan (AWKUM), Mardan, Pakistan \\ ${ }^{4}$ Department of Mathematics, Faculty of Science, King Khalid University, Abha 61413, Saudi Arabia \\ ${ }^{5}$ Department of Mathematics, Faculty of Science, AL-Azhar University, Assiu, 71516, Egypt \\ ${ }^{6}$ Department of Mathematics, Faculty of Arts and Sciences, Cankaya University, Ankara 06530, Turkey \\ ${ }^{7}$ Institute of Space Sciences, Magurele-Bucharest, Romania \\ Correspondence should be addressed to Hassan Khan; hassanmath@awkum.edu.pk
}

Received 2 April 2020; Revised 22 June 2020; Accepted 1 July 2020; Published 28 July 2020

Academic Editor: Dimitri Volchenkov

Copyright (c) 2020 Haobin Liu et al. This is an open access article distributed under the Creative Commons Attribution License, which permits unrestricted use, distribution, and reproduction in any medium, provided the original work is properly cited.

In this paper, the fractional view analysis of the Keller-Segal equations with sensitivity functions is presented. The Caputo operator has been used to pursue the present research work. The natural transform is combined with the homotopy perturbation method, and a new scheme for implementation is derived. The modified established method is named as the homotopy perturbation transform technique. The derived results are compared with the solution of the Laplace Adomian decomposition technique by using the systems of fractional Keller-Segal equations. The solution graphs and the table have shown that the obtained results coincide with the solution of the Laplace Adomian decomposition method. Fractionalorder solutions are determined to confirm the reliability of the current method. It is observed that the solutions at various fractional orders are convergent to an integer-order solution of the problems. The suggested procedure is very attractive and straight forward and therefore can be modified to solve high nonlinear fractional partial differential equations and their systems.

\section{Introduction}

Fractional differential equations (FDEs) are the generalizations of the standard integer-order differential equations. FDEs have gained much attention in the recent decades, as they are broadly used in several areas to analyze different processes, such as the processing of the signal, control theory, identification of the system, fluid flow, biomathematics, and other fields [1-3]. FDEs have been implemented in the modeling of the description of unification of diffusion, fractional random walk, and systems of both diffusive and subdiffusive. It is also an investigation that in many physical phenomena, experiments have proved that the solution of fractional-order DEs is in good agreement with experimental data of any physical phenomena to integer-order DEs. For example, the noninteger differential equations (DEs) can model some physical phenomena more effectively such as delineates memory, heredity effects, properties of different materials, and internal friction process [4-9].

The importance of FDEs is found in the literature because it can model most of the physical phenomena in science and engineering more accurately as compared to integer-order models [10-12], and therefore the researchers have shown much interest to study fractional calculus and FDEs during the last decades. Because of the significance of FDEs, the mathematicians are working to develop many useful and powerful numerical and analytical techniques to determine the actual or approximate solutions of the 
targeted problems [13-15]. Many other powerful and efficient techniques have been suggested to obtain the exact or analytical solutions for FDEs. For instance, these techniques include the Laplace Adomian decomposition method (LADM) [16-18], Chebyshev wavelet methods (CWM) [19], collocation-shooting method [20], power series methods (PSM) [21], fractional Bernstein polynomials along with shooting method [22], fractional-Legendre spectral Galerkin method [23], variational iterative transform method (VITM) [24], homotopy perturbation transform method (HPTM) [25-27], homotopy analysis transform method (HATM) [28, 29], reduced differential transform method (RDTM) [30, 31], finite element technique (FET) [32], finite difference technique (FDT) [33], and $q$-homotopy analysis transform method (q-HATM) [34]. Based on these techniques, a wide range of FDEs have been analyzed.

The most important cell motion which can be used as a response to the gradient of a chemical compound is known as chemotaxis. It has an important contribution in the population cell number and growth of biology. E. Keller and L. Segal suggested the first mathematical equation of chemotaxis in 1970. They displayed parabolic schemes to describe the method of aggregating a cell slime mold depending on a molecule attraction [35]. In this article, we considered the coupled time-fractional Keller-Segel equation [36-39]:

$$
\begin{array}{r}
D_{\Psi}^{\gamma} \mu(\zeta, \Psi)-a \frac{\partial^{2} \mu(\zeta, \Psi)}{\partial \zeta^{2}}+\frac{\partial}{\partial \zeta}\left\{\mu(\zeta, \Psi) \frac{\partial[\chi \rho(\zeta, \psi)]}{\partial \zeta}\right\}=0 \\
D_{\Psi}^{\gamma} \rho(\zeta, \Psi)-b \frac{\partial^{2} \rho(\zeta, \Psi)}{\partial \zeta^{2}}-c \mu(\zeta, \Psi)+d \rho(\zeta, \Psi)=0 .
\end{array}
$$

With initial conditions,

$$
\left\{\begin{array}{l}
\mu(\zeta, \Psi)=\mu_{0}(\zeta) \\
\rho(\zeta, \Psi)=\rho_{0}(\zeta)
\end{array}\right.
$$

where the unidentified function $\mu(\zeta, \Psi)$ defines the concentrations of amoebae, the chemical concentration is denoted by $\rho(\zeta, \Psi)$, and the chemotactic is defined by the partial derivative of $\partial / \partial \zeta\{\mu(\zeta, \Psi) \partial[\chi \rho(\zeta, \psi)] / \partial \zeta\}, \chi(\rho)$ is the sensitivity function. Some other types of sensitivity functions can be observed in the coupled time-fractional K-S chemotactic model in [40] and also in the K-S model with a logic sensitivity function and small diffusivity [41]. Also, singular sensitivity can be seen in the system of the two-dimensional $\mathrm{K}-\mathrm{S}$ system in $[36,42,43]$. The K-S model has been extensively analyzed recently. For example, Kamel et.al. have used LADM for the solution of the S-K equation [37]. Bournaveas [38] gave the one-dimensional Keller-Segel model with a fractional cell diffusion. Zayernouri [39] formed a fractional class of implicit Adams-Moulton and explicit Adams-Bashforth techniques.

The HPM is mixed with the natural transform method to generate a highly effective method to handle the solution of several nonlinear problems and is known as the homotopy perturbation transform method (HPTM). HPTM generates a convergent series form solution that converges to the exact solution of the problems and provides closed-form solutions. The proposed method can combine two important techniques to achieve an effective solution for nonlinear equations. Gorbhani first proposed the use of He's polynomial in the nonlinear terms [44, 45]. It should be remembered that the HPTM is implemented without any flexibility or restrictive assumptions or transforms and round off free error. Many authors have solved fractional-order diffusion equations [46], partial differential equations [47, 48], and wave-like equations [49] by using linear and nonlinear problems with the help of HPTM.

In the current research paper, HPTM is implemented to solve fractional-order Keller-Segel equations. The solutions achieved through the suggested technique are straightforward and simple. The quality of the current method is appropriate to provide the analytical results to the given examples. The HPTM solutions are shown to be in close contact with the solutions of other existing techniques.

\section{Preliminary Concepts}

Definition 1. Let $g \in C_{\beta}$ and $\beta \geq-1$, and then the Riemann-Liouville integral of order $\gamma$ is given by [1-3]

$$
J_{\Psi}^{\gamma} g(\zeta, \Psi)=\frac{1}{\Gamma(\gamma)} \int_{0}^{\Psi}(\Psi-\theta)^{\gamma-1} g(\zeta, \theta) d \theta, \Psi>0 .
$$

Definition 2. Let $g \in \mathbb{C}_{\beta}$ and $\beta \geq-1$, and then the Caputo definition of the fractional derivative of order $\gamma$ if $m-1<\gamma \leq m$ with $m \in \mathbb{N}$ is described as [1-3]

$$
D_{\Psi}^{\gamma} g(\Psi)=\left\{\begin{array}{l}
\frac{d^{m} g(\Psi)}{d \Psi^{m}}, \quad \gamma=m \in N, \\
\frac{1}{\Gamma(m-\gamma)} \int_{0}^{\Psi}(\Psi-\theta)^{m-\gamma-1} g^{(m)}(\theta) d \theta, \\
m-1<\gamma<m, \quad m \in N .
\end{array}\right.
$$

Remark 1. Some basic properties are given below [1-3]:

$$
\begin{aligned}
& D_{\zeta}^{\gamma} I_{\zeta}^{\gamma} g(\zeta)=g(\zeta), I^{\gamma} \zeta^{\lambda}=\frac{\Gamma(\lambda+1)}{\Gamma(\gamma+\lambda+1)} \zeta^{\gamma+\lambda}, \quad \gamma>0, \lambda>-1, \zeta>0, \\
& D_{\zeta}^{\gamma} I_{\zeta}^{\gamma} g(\zeta)=g(\zeta)-\sum_{k=0}^{m} g^{(k)}\left(0^{+}\right) \frac{\zeta^{k}}{k !}, \quad \text { for } \zeta>0 .
\end{aligned}
$$

Definition 3. The natural transform $f(\Psi)$ of the function $N[f(\Psi)]$ for $\Psi \in R$ is defined as [50] 


$$
\begin{aligned}
& N[f(\Psi)]=\mathscr{G}(s, u)=\int_{-\infty}^{\infty} e^{-s \Psi} f(\Psi) d \Psi ; \\
& s, u \in(-\infty, \infty), \\
& N[f(\Psi) Q(\Psi)]=N^{+}[f(\Psi)]=\mathscr{G}^{+}(s, u) \\
&=\int_{0}^{\infty} e^{-s \Psi} f(\Psi) d \Psi ; \\
& s, u \in(0, \infty), \quad \text { and } \quad \Psi \in R .
\end{aligned}
$$

Theorem 1. The natural transform of the fractional derivative of the function in the Riemann-Liouville sense is given as [50]

$$
\begin{aligned}
N^{+}\left[D^{\gamma} f(\Psi)\right]= & \mathscr{G}_{\gamma}(s, u)=\frac{s^{\gamma}}{u^{\gamma}} \mathscr{G}(s, u) \\
& -\sum_{j=0}^{m-1} \frac{s^{j}}{u^{\gamma-j}}\left[D^{\gamma-j-1} f(\Psi)\right]_{\Psi=0}, \quad \ell-1 \leq \gamma<\ell .
\end{aligned}
$$

Theorem 2. The natural transform of the fractional derivative of the function in the Caputo sense is given as [50]

$$
\begin{aligned}
N^{+}\left[{ }^{c} D^{\gamma} f(\Psi)\right]= & \mathscr{G}_{\gamma}^{c}(s, u)=\frac{s^{\gamma}}{u^{\gamma}} \mathscr{G}(s, u) \\
& -\sum_{j=0}^{\ell-1} \frac{s^{\gamma-(j+1)}}{u^{\gamma-j}}\left[D^{j} f(\Psi)\right]_{\Psi=0}, \quad \ell-1 \leq \gamma<\ell .
\end{aligned}
$$

\section{Implementation of HPTM [51]}

To explain the fundamental concept of this technique, we consider a particular fractional-order nonlinear partial differential nonhomogeneous equation:

$$
D_{\Psi}^{\gamma} \mu(\zeta, \Psi)+\mathscr{R} \mu(\zeta, \Psi)+\mathcal{N} \mu(\zeta, \Psi)=g(\zeta, \Psi), \quad 1<\gamma \leq 2 .
$$

With initial conditions,

$$
\mu(\zeta, 0)=h(\zeta), \quad \mu_{\Psi}(\zeta, 0)=f(\zeta),
$$

where the fractional derivative in equation (9) is defined in the Caputo sense. The operators $\mathscr{R}$ and $\mathcal{N}$ describe the linear and nonlinear operators, respectively, and $g(\zeta, \Psi)$ is the source term.

Using natural transformation in equation (9), we get [51]

$$
N\left[D_{\Psi}^{\gamma} \mu(\zeta, \Psi)\right]+N[\mathscr{R} \mu(\zeta, \Psi)]+N[\mathcal{N} \mu(\zeta, \Psi)]=N[g(\zeta, \Psi)] .
$$

With the help of the fractional derivative natural property, we have

$$
\begin{aligned}
\mathrm{N}[\mu(\zeta, \Psi)]= & \frac{h(\zeta)}{s}+\frac{f(\zeta)}{s^{2}}+\frac{u^{\gamma}}{s^{\gamma}} \mathrm{N}[g(\zeta, \Psi)] \\
& -\frac{u^{\gamma}}{s^{\gamma}} \mathrm{N}[\mathscr{R} \mu(\zeta, \Psi)]-\frac{u^{\gamma}}{s^{\gamma}} \mathrm{N}[\mathcal{N} \mu(\zeta, \Psi)] .
\end{aligned}
$$

Taking the inverse natural transformation of equation (12), we get

$$
\mu(\zeta, \Psi)=\mathscr{G}(\zeta, \Psi)-N^{-1}\left\{\frac{u^{\gamma}}{s^{\gamma}} N[\mathscr{R} \mu(\zeta, \Psi)+\mathscr{N} \mu(\zeta, \Psi)]\right\} .
$$

Using the HPTM procedure, the solution is expressed as

$$
\mu(\zeta, \Psi)=\sum_{m=0}^{\infty} p^{m} \mu_{m}(\zeta, \Psi) .
$$

The nonlinear term can be decomposed as

$$
\mathcal{N} \mu(\zeta, \Psi)=\sum_{m=0}^{\infty} p^{m} \mathscr{H}_{m}(\zeta, \Psi) .
$$

Few He's polynomials $\mathscr{H}_{m}(u)[35,40]$ are described by

$$
\mathscr{H}_{\ell}\left(\mu_{0}, \mu_{1}, \ldots, \mu_{\ell}\right)=\frac{1}{\ell !} \frac{\partial^{\ell}}{\partial p^{\ell}}\left[N\left(\sum_{i=0}^{\infty} p^{i} \mu_{i}\right)\right]_{p=0}, \quad \ell=0,1,2,3, \ldots
$$

Utilizing equations (14) and (15) in equation (13), we have

$$
\begin{aligned}
\sum_{\ell=0}^{\infty} p^{\ell} \mu_{\ell}(\zeta, \Psi)= & \mathscr{G}(\zeta, \Psi)-p\left(\left\{\frac { u ^ { \gamma } } { s ^ { \gamma } } \mathrm { N } \left[\mathscr{R} \sum_{\ell=0}^{\infty} p^{\ell} \mu_{\ell}(\zeta, \Psi)\right.\right.\right. \\
& \left.\left.\left.+\sum_{\ell=0}^{\infty} p^{\ell} \mathscr{H}_{\ell}(\zeta, \Psi)\right]\right\}\right) .
\end{aligned}
$$

Using He's polynomials in HPTM and comparing the coefficient of power $p$, we get

$$
\begin{aligned}
& p^{0}: \mu_{0}(\zeta, \Psi)=\mathscr{G}(\zeta, \Psi), \\
& p^{1}: \mu_{1}(\zeta, \Psi)=-N^{-1}\left\{\frac{u^{\gamma}}{s^{\gamma}} N\left[\mathscr{R} \mu_{0}(\zeta, \Psi)+\mathscr{H}_{0} \mu\right]\right\}, \\
& p^{2}: \mu_{2}(\zeta, \Psi)=-N^{-1}\left\{\frac{u^{\gamma}}{s^{\gamma}} N\left[\mathscr{R} \mu_{1}(\zeta, \Psi)+\mathscr{H}_{1} \mu\right]\right\}, \\
& p^{3}: \mu_{3}(\zeta, \Psi)=-N^{-1}\left\{\frac{u^{\gamma}}{s^{\gamma}} N\left[\mathscr{R} \mu_{2}(\zeta, \Psi)+\mathscr{H}_{2} \mu\right]\right\} .
\end{aligned}
$$

The remaining of the $\mu_{\ell}(\zeta, \Psi)$ components can be totally obtained, and the sequence result is thus fully determined.

Finally, we calculate the analytical result of $\mu(\zeta, \Psi)$ : 


$$
\mu(\zeta, \Psi)=\lim _{N \longrightarrow \infty} \sum_{\ell=0}^{N} \mu_{\ell}(\zeta, \Psi) .
$$

Generally, the solutions of the above series converge very quickly.

\section{Numerical Examples}

Example 1. The Keller-Segal equations with a fractional derivative are given as [37-39]

$$
\begin{array}{r}
D_{\Psi}^{\gamma} \mu(\zeta, \Psi)-a \frac{\partial^{2} \mu(\zeta, \Psi)}{\partial \zeta^{2}}+\frac{\partial}{\partial \zeta}\left\{\mu(\zeta, \Psi) \frac{\partial[\chi \rho(\zeta, \psi)]}{\partial \zeta}\right\}=0 \\
D_{\Psi}^{\gamma} \rho(\zeta, \Psi)-b \frac{\partial^{2} \rho(\zeta, \Psi)}{\partial \zeta^{2}}-c \mu(\zeta, \Psi)+d \rho(\zeta, \Psi)=0 .
\end{array}
$$

Subjecting to the initial solutions, we get

$$
\begin{aligned}
& \mu(\zeta, 0)=m e^{-\zeta^{2}}, \\
& \rho(\zeta, 0)=n e^{-\zeta^{2}} .
\end{aligned}
$$

Taking natural transformation of equation (20), we get

$$
\begin{aligned}
N\left[\frac{\partial^{\gamma} \mu(\zeta, \Psi)}{\partial \Psi^{\gamma}}\right] & =N\left[a \frac{\partial^{2} \mu(\zeta, \Psi)}{\partial \zeta^{2}}-\frac{\partial}{\partial \zeta}\left(\mu(\zeta, \Psi) \frac{\partial[\chi \rho(\zeta, \psi)]}{\partial \zeta}\right)\right], \\
N\left[\frac{\partial^{\gamma} \rho(\zeta, \Psi)}{\partial \Psi^{\gamma}}\right] & =N\left[b \frac{\partial^{2} \rho(\zeta, \Psi)}{\partial \zeta^{2}}+c \mu(\zeta, \Psi)-d \rho(\zeta, \Psi)\right], \\
\frac{s^{\gamma}}{u^{\gamma}} N\{\mu(\zeta, \Psi)\}-\frac{s^{\gamma-1}}{u^{\gamma}} \mu(\zeta, 0) & =N\left[a \frac{\partial^{2} \mu(\zeta, \Psi)}{\partial \zeta^{2}}-\frac{\partial}{\partial \zeta}\left(\mu(\zeta, \Psi) \frac{\partial[\chi \rho(\zeta, \psi)]}{\partial \zeta}\right)\right], \\
\frac{s^{\gamma}}{u^{\gamma}} N\{\rho(\zeta, \Psi)\}-\frac{s^{\gamma-1}}{u^{\gamma}} \rho(\zeta, 0) & =N\left[b \frac{\partial^{2} \rho(\zeta, \Psi)}{\partial \zeta^{2}}+c \mu(\zeta, \Psi)-d \rho(\zeta, \Psi)\right] .
\end{aligned}
$$

Simplifying the above equation, we get

$$
\begin{aligned}
& N[\mu(\zeta, \Psi)]=\frac{1}{s}[\mu(\zeta, 0)]+\frac{u^{\gamma}}{s^{\gamma}} N\left(a \frac{\partial^{2} \mu(\zeta, \Psi)}{\partial \zeta^{2}}-\frac{\partial}{\partial \zeta}\left\{\mu(\zeta, \Psi) \frac{\partial[\chi \rho(\zeta, \psi)]}{\partial \zeta}\right\}\right), \\
& N[\rho(\zeta, \Psi)]=\frac{1}{s}[\rho(\zeta, 0)]+\frac{u^{\gamma}}{s^{\gamma}} N\left[b \frac{\partial^{2} \rho(\zeta, \Psi)}{\partial \zeta^{2}}+c \mu(\zeta, \Psi)-d \rho(\zeta, \Psi)\right] .
\end{aligned}
$$

Using inverse natural transform, we have

$$
\begin{aligned}
& \mu(\zeta, \Psi)=\mu(\zeta, 0)-\mathrm{N}^{-}\left\{\frac{u^{\gamma}}{s^{\gamma}} \mathrm{N}\left[a \frac{\partial^{2} \mu(\zeta, \Psi)}{\partial \zeta^{2}}-\frac{\partial}{\partial \zeta}\left(\mu(\zeta, \Psi) \frac{\partial(\chi \rho(\zeta, \psi))}{\partial \zeta}\right)\right]\right\}, \\
& \rho(\zeta, \Psi)=\rho(\zeta, 0)-\mathrm{N}^{-} \partial^{2} \rho(\zeta, \Psi)\left\{\frac{u^{\gamma}}{s^{\gamma}} \mathrm{N}\left[b \frac{\partial^{2} \rho(\zeta, \Psi)}{\partial \zeta^{2}}+c \mu(\zeta, \Psi)-d \rho(\zeta, \Psi)\right]\right\} .
\end{aligned}
$$


Then, the chemotactic term $\partial / \partial \zeta\{\mu(\zeta, \Psi) \partial[\chi \rho(\zeta, \psi)] /$ Now, implementing HPM, we get $\partial \zeta\}=0$.

$$
\begin{aligned}
& \sum_{\ell=0}^{\infty} p^{\ell} \mu_{\ell}(\zeta, \Psi)=\mathrm{me}^{-\zeta^{2}}+p\left(\mathrm{~N}^{-}\left\{\frac{u^{\gamma}}{s^{\gamma}} \mathrm{N}\left[a \sum_{\ell=0}^{\infty} p^{\ell} \frac{\partial^{2} \mu_{\ell}(\zeta, \Psi)}{\partial \zeta^{2}}\right]\right\}\right), \\
& \sum_{\ell=0}^{\infty} p^{\ell} \rho_{\ell}(\zeta, \Psi)=\mathrm{ne}^{-\zeta^{2}}+p\left(\mathrm { N } ^ { - } \left\{\begin{array}{l}
\left.\frac{u^{\gamma}}{s^{\gamma}} \mathrm{N}\left[\begin{array}{l}
b \sum_{\ell=0}^{\infty} p^{\ell} \frac{\partial^{2} \rho_{\ell}(\zeta, \Psi)}{\partial \zeta^{2}}+c \sum_{\ell=0}^{\infty} p^{\ell} \mu_{\ell}(\zeta, \Psi) \\
-d \sum_{\ell=0}^{\infty} p^{\ell} \rho_{\ell}(\zeta, \Psi)
\end{array}\right\}\right)
\end{array}\right.\right.
\end{aligned}
$$

By comparing the coefficients of powers $p$, we get

$$
\begin{aligned}
p^{0}: \mu_{0}(\zeta, \Psi)= & m e^{-\zeta^{2}}, \\
p^{0}: \rho_{0}(\zeta, \Psi)= & n e^{-\zeta^{2}}, \\
p^{1}: \mu_{1}(\zeta, \Psi)= & \mathrm{N}^{-}\left\{\frac{u^{\gamma}}{s^{\gamma}} \mathrm{N}\left[a \frac{\partial^{2} \mu_{0}(\zeta, \Psi)}{\partial \zeta^{2}}\right]\right\}, \\
\mu_{1}(\zeta, \Psi)= & 2 \mathrm{am}\left(2 \zeta^{2}-1\right) e^{-\zeta^{2}} \frac{\Psi^{\gamma}}{\Gamma(\gamma+1)}, \\
p^{1}: \rho_{1}(\zeta, \Psi)= & \mathrm{N}^{-}\left\{\frac{u^{\gamma}}{s^{\gamma}} \mathrm{N}\left[b \frac{\partial^{2} \rho_{0}(\zeta, \Psi)}{\partial \zeta^{2}}+c \mu_{0}(\zeta, \Psi)-d \rho_{0}(\zeta, \Psi)\right]\right\}, \\
\rho_{1}(\zeta, \Psi)= & \left\{2 b n\left(2 \zeta^{2}-1\right)+(\mathrm{cm}-\mathrm{dn})\right\} e^{-\zeta^{2}} \frac{\Psi \gamma}{\Gamma(\gamma+1)}, \\
& +\left[\left(2-4 \zeta^{2}-1\right)(\mathrm{cm}+d n)-2 c a\left(2 m \zeta^{2}-m\right)\right] e^{-\zeta^{2}} \frac{\Psi^{2 \gamma}}{\Gamma(2 \gamma+1)} . \\
p^{2}: \mu_{2}(\zeta, \Psi)= & \mathrm{N}^{-}\left\{\frac{u^{\gamma}}{s^{\gamma}} \mathrm{N}\left[a \frac{\partial^{2} \mu_{1}(\zeta, \Psi)}{\partial \zeta^{2}}\right]\right\}, \\
\rho_{2}(\zeta, \Psi)= & b\left[-24 \mathrm{bn}-4 \mathrm{bn}\left(2 m^{2}-1\right)+\left(8 b \zeta^{2}-2 b d n\right)\left(2 \zeta^{2}-1\right)\right] e^{-\zeta^{2}} \frac{\Psi^{2 \gamma}}{\Gamma(2 \gamma+1)} \\
\mu_{2}(\zeta, \Psi)= & 4 a^{2}\left(3 m-6 m \zeta^{2}-6 \mathrm{am} \zeta^{2}+4 m \zeta^{3}\right) e^{-\zeta^{2}} \frac{\Psi^{2 \gamma}}{\Gamma(2 \gamma+1)}, \\
p^{2}: \rho_{2}(\zeta, \Psi)= & \mathrm{N}^{-}\left\{\frac{u^{\gamma}}{s^{\gamma}} \mathrm{N}\left[b \frac{\partial^{2} \rho_{1}(\zeta, \Psi)}{\partial \zeta^{2}}+c \mu_{1}(\zeta, \Psi)-d \rho_{1}(\zeta, \Psi)\right]\right\}, \\
&
\end{aligned}
$$


The series form of equation (20) is given by The HPTM solution is given as

$$
\begin{aligned}
\mu(\zeta, \Psi)= & \sum_{\ell=0}^{\infty} \mu_{\ell}(\zeta, \Psi)=\mu_{0}(\zeta, \Psi)+\mu_{1}(\zeta, \Psi)+\mu_{2}(\zeta, \Psi) \\
& +\mu_{3}(\zeta, \Psi)+\cdots, \\
\rho(\zeta, \Psi)= & \sum_{\ell=0}^{\infty} \rho_{\ell}(\zeta, \Psi)=\rho_{0}(\zeta, \Psi)+\rho_{1}(\zeta, \Psi)+\rho_{2}(\zeta, \Psi) \\
& +\rho_{3}(\zeta, \Psi)+\cdots .
\end{aligned}
$$

$$
\begin{aligned}
\mu(\zeta, \Psi)= & m e^{-\zeta^{2}}+2 \operatorname{am}\left(2 \zeta^{2}-1\right) e^{-\zeta^{2}} \frac{\Psi^{\gamma}}{\Gamma(\gamma+1)}+4 a^{2}\left(3 m-6 m \zeta^{2}-6 \mathrm{am} \zeta^{2}+4 m \zeta^{3}\right) e^{-\zeta^{2}} \frac{\Psi^{2 \gamma}}{\Gamma(2 \gamma+1)}+\cdots \\
\rho(\zeta, \Psi)= & n e^{-\zeta^{2}}+\left\{2 b n\left(2 \zeta^{2}-1\right)+(c m-d n)\right\} e^{-\zeta^{2}} \frac{\Psi^{\gamma}}{\Gamma(\gamma+1)}+b\left\{-24 b n-4 b n\left(2 m^{2}-1\right)+\left(8 b \zeta^{2}-2 b d n\right)\left(2 \zeta^{2}-1\right)\right\} e^{-\zeta^{2}} \\
& \cdot \frac{\Psi^{2 \gamma}}{\Gamma(2 \gamma+1)}+\left\{\left(2-4 \zeta^{2}-1\right)(\mathrm{cm}+d n)-2 c a\left(2 m \zeta^{2}-m\right)\right\} e^{-\zeta^{2}} \frac{\Psi^{2 \gamma}}{\Gamma(2 \gamma+1)}+\cdots
\end{aligned}
$$

The series solutions are obtained by using the numerical values $d=0.8, m=160, b=3, n=120, a=0.5$, and $c=1$ :

$$
\begin{aligned}
\mu(\zeta, \Psi)= & 160 e^{-\zeta^{2}}+160\left(2 \zeta^{2}-1\right) e^{-\zeta^{2}} \frac{\Psi^{\gamma}}{\Gamma(\gamma+1)}+5\left(96-384 \zeta^{2}+128 \zeta^{3}\right) e^{-\zeta^{2}} \frac{\Psi^{2 \gamma}}{\Gamma(2 \gamma+1)} \\
& +5\left(252-24 \zeta^{2}+128 \zeta^{3}+175 \zeta^{4}\right) e^{-\zeta^{2}} \frac{\Psi^{3 \gamma}}{\Gamma(3 \gamma+1)}+\cdots \\
\rho(\zeta, \Psi)= & 120 e^{-\zeta^{2}}+\left[320\left(2 \zeta^{2}-1\right)-64\right] e^{-\zeta^{2}} \frac{\Psi^{\gamma}}{\Gamma(\gamma+1)}+\left[3450-7680 \zeta^{2}-1920\left(2 \zeta^{2}-1\right)\right. \\
& \left.-7680 \zeta+12 \zeta^{2}\left(320\left(2 \zeta^{2}-1\right)+64\right)\right] \\
& +5\left(192 \zeta-128 \zeta^{3}\right)-2\left\{320-\zeta\left[160\left(2 \zeta^{2}-1\right)+32\right]\right\} \\
& \cdot e^{-\zeta^{2}} \frac{\Psi^{2 \gamma}}{\Gamma(2 \gamma+1)}\left\{5\left(1360-215 \zeta^{2}-28\left(8 \zeta^{2}+7 \zeta^{3}-2\right)-3045 \zeta+8 \zeta^{2}\left(8 \zeta^{2}+7 \zeta^{3}-2\right)\right)\right. \\
& \left.+\left(123 \zeta-840 \zeta^{3}\right)-0.9\left(1280 \zeta-2 \zeta\left[160\left(8 \zeta^{2}+7 \zeta^{3}-2\right)+64\right]\right)\right\} \\
& \cdot e^{-\zeta^{2}} \frac{\Psi^{3 \gamma}}{\Gamma(3 \gamma+1)}+\cdots
\end{aligned}
$$

Example 2. The Keller-Segal equations with a fractional derivative are given as [37-39] 


$$
\begin{gathered}
D_{\Psi}^{\gamma} \mu(\zeta, \Psi)-a \frac{\partial^{2} \mu(\zeta, \Psi)}{\partial \zeta^{2}}+\frac{\partial \mu(\zeta, \Psi)}{\partial \zeta} \frac{\partial \rho(\zeta, \psi)}{\partial \zeta} \\
-\mu(\zeta, \Psi) \frac{\partial^{2} \rho(\zeta, \psi)}{\partial \zeta^{2}}=0, \\
D_{\Psi}^{\gamma} \rho(\zeta, \Psi)-b \frac{\partial^{2} \rho(\zeta, \Psi)}{\partial \zeta^{2}}-c \mu(\zeta, \Psi)+d \rho(\zeta, \Psi)=0 .
\end{gathered}
$$

With the initial conditions:

$$
\begin{aligned}
& \mu(\zeta, 0)=m e^{-\zeta^{2}}, \\
& \rho(\zeta, 0)=n e^{-\zeta^{2}} .
\end{aligned}
$$

Applying natural transformation to equation (30), we get

$$
\begin{aligned}
N\left\{\frac{\partial^{\gamma} \mu(\zeta, \Psi)}{\partial \Psi^{\gamma}}\right\} & =N\left[a \frac{\partial^{2} \mu(\zeta, \Psi)}{\partial \zeta^{2}}-\frac{\partial \mu(\zeta, \Psi)}{\partial \zeta} \frac{\partial \rho(\zeta, \psi)}{\partial \zeta}+\mu(\zeta, \Psi) \frac{\partial^{2} \rho(\zeta, \psi)}{\partial \zeta^{2}}\right] \\
N\left\{\frac{\partial^{\gamma} \rho(\zeta, \Psi)}{\partial \Psi^{\gamma}}\right\} & =N\left[b \frac{\partial^{2} \rho(\zeta, \Psi)}{\partial \zeta^{2}}+c \mu(\zeta, \Psi)-d \rho(\zeta, \Psi)\right] \\
\frac{s^{\gamma}}{u^{\gamma}} N\{\mu(\zeta, \Psi)\}-\frac{s^{\gamma-1}}{u^{\gamma}} \mu(\zeta, 0) & =N\left[a \frac{\partial^{2} \mu(\zeta, \Psi)}{\partial \zeta^{2}}-\frac{\partial \mu(\zeta, \Psi)}{\partial \zeta} \frac{\partial \rho(\zeta, \psi)}{\partial \zeta}+\mu(\zeta, \Psi) \frac{\partial^{2} \rho(\zeta, \psi)}{\partial \zeta^{2}}\right], \\
\frac{s^{\gamma}}{u^{\gamma}} N\{\rho(\zeta, \Psi)\}-\frac{s^{\gamma-1}}{u^{\gamma}} \rho(\zeta, 0) & =N\left[b \frac{\partial^{2} \rho(\zeta, \Psi)}{\partial \zeta^{2}}+c \mu(\zeta, \Psi)-d \rho(\zeta, \Psi)\right] .
\end{aligned}
$$

Simplifying the above equation, we get

$$
\begin{aligned}
& N\{\mu(\zeta, \Psi)\}=\frac{1}{s}\{\mu(\zeta, 0)\}+\frac{u^{\gamma}}{s^{\gamma}} N\left[a \frac{\partial^{2} \mu(\zeta, \Psi)}{\partial \zeta^{2}}-\frac{\partial \mu(\zeta, \Psi)}{\partial \zeta} \frac{\partial \rho(\zeta, \psi)}{\partial \zeta}+\mu(\zeta, \Psi) \frac{\partial^{2} \rho(\zeta, \psi)}{\partial \zeta^{2}}\right] \\
& N\{\rho(\zeta, \Psi)\}=\frac{1}{s}\{\rho(\zeta, 0)\}+\frac{u^{\gamma}}{s^{\gamma}} N\left[b \frac{\partial^{2} \rho(\zeta, \Psi)}{\partial \zeta^{2}}+c \mu(\zeta, \Psi)-d \rho(\zeta, \Psi)\right]
\end{aligned}
$$

Using inverse natural transform, we have

$$
\begin{aligned}
& \mu(\zeta, \Psi)=\mu(\zeta, 0)-N^{-}\left[\frac{u^{\gamma}}{s^{\gamma}} N\left[a \frac{\partial^{2} \mu(\zeta, \Psi)}{\partial \zeta^{2}}-\frac{\partial \mu(\zeta, \Psi)}{\partial \zeta} \frac{\partial \rho(\zeta, \psi)}{\partial \zeta}+\mu(\zeta, \Psi) \frac{\partial^{2} \rho(\zeta, \psi)}{\partial \zeta^{2}}\right]\right] \\
& \rho(\zeta, \Psi)=\rho(\zeta, 0)-N^{-}\left[\frac{u^{\gamma}}{s^{\gamma}} N\left[b \frac{\partial^{2} \rho(\zeta, \Psi)}{\partial \zeta^{2}}+c \mu(\zeta, \Psi)-d \rho(\zeta, \Psi)\right]\right] .
\end{aligned}
$$

Now, implementing HPM, we get

$$
\begin{aligned}
& \sum_{\ell=0}^{\infty} p^{\ell} \mu_{\ell}(\zeta, \Psi)=\mathrm{me}^{-\zeta^{2}}+p\left[N^{-}\left[\frac{u^{\gamma}}{s^{\gamma}} N\left[a \sum_{\ell=0}^{\infty} p^{\ell} \frac{\partial^{2} \mu_{\ell}(\zeta, \Psi)}{\partial \zeta^{2}}-\sum_{\ell=0}^{\infty} p^{\ell} \mathscr{H}_{\ell}+\sum_{\ell=0}^{\infty} p^{\ell} \mathscr{H}_{\ell}^{\prime}\right]\right]\right] \\
& \sum_{\ell=0}^{\infty} p^{\ell} \rho_{\ell}(\zeta, \Psi)=\mathrm{ne}^{-\zeta^{2}}+p\left[N^{-}\left[\frac{u^{\gamma}}{s^{\gamma}} N\left[b \sum_{\ell=0}^{\infty} p^{\ell} \frac{\partial^{2} \rho_{\ell}(\zeta, \Psi)}{\partial \zeta^{2}}+c \sum_{\ell=0}^{\infty} p^{\ell} \mu_{\ell}(\zeta, \Psi)-d \sum_{\ell=0}^{\infty} p^{\ell} \rho_{\ell}(\zeta, \Psi)\right]\right]\right]
\end{aligned}
$$


where $\mathscr{H}_{\ell}$ and $\mathscr{H}_{\ell}^{\prime}$ are He's polynomials that show the nonlinear terms. So, representing He's polynomials for $\mathscr{H}_{\ell}(\mu)$, we find that

$$
\begin{aligned}
\sum_{\ell=0}^{\infty} p^{\ell} \mathscr{H}_{\ell} & =\frac{\partial \mu(\zeta, \Psi)}{\partial \zeta} \frac{\partial \rho(\zeta, \psi)}{\partial \zeta}, \\
\mathscr{H}_{0} & =\frac{\partial \mu_{0}(\zeta, \Psi)}{\partial \zeta} \frac{\partial \rho_{0}(\zeta, \psi)}{\partial \zeta}, \\
\mathscr{H}_{1} & =\frac{\partial \mu_{0}(\zeta, \Psi)}{\partial \zeta} \frac{\partial \rho_{1}(\zeta, \psi)}{\partial \zeta}+\frac{\partial \mu_{1}(\zeta, \Psi)}{\partial \zeta} \frac{\partial \rho_{0}(\zeta, \psi)}{\partial \zeta}, \\
\mathscr{H}_{2} & =\frac{\partial \mu_{0}(\zeta, \Psi)}{\partial \zeta} \frac{\partial \rho_{2}(\zeta, \psi)}{\partial \zeta}+\frac{\partial \mu_{1}(\zeta, \Psi)}{\partial \zeta} \frac{\partial \rho_{1}(\zeta, \psi)}{\partial \zeta}+\frac{\partial \mu_{2}(\zeta, \Psi)}{\partial \zeta} \frac{\partial \rho_{0}(\zeta, \psi)}{\partial \zeta}
\end{aligned}
$$

For $\mathscr{H}_{l}^{\prime}(\mu)$, we find that

$$
\begin{aligned}
\sum_{\ell=0}^{\infty} p^{\ell} \mathscr{H}_{l}^{\prime} & =\mu(\zeta, \Psi) \frac{\partial^{2} \rho(\zeta, \psi)}{\partial \zeta^{2}}, \\
\mathscr{H}_{0}^{\prime} & =\mu_{0}(\zeta, \Psi) \frac{\partial^{2}\left[\rho_{0}(\zeta, \psi)\right]}{\partial \zeta^{2}}, \\
\mathscr{H}_{l}^{\prime} & =\mu_{0}(\zeta, \Psi) \frac{\partial^{2}\left[\rho_{0}(\zeta, \psi)\right]}{\partial \zeta^{2}}+\mu_{1}(\zeta, \Psi) \frac{\partial^{2}\left[\rho_{0}(\zeta, \psi)\right]}{\partial \zeta^{2}}, \\
\mathscr{H}_{2}^{\prime} & =\mu_{0}(\zeta, \Psi) \frac{\partial^{2}\left[\rho_{2}(\zeta, \psi)\right]}{\partial \zeta^{2}}+\mu_{1}(\zeta, \Psi) \frac{\partial^{2}\left[\rho_{1}(\zeta, \psi)\right]}{\partial \zeta^{2}}+\mu_{2}(\zeta, \Psi) \frac{\partial^{2}\left[\rho_{0}(\zeta, \psi)\right]}{\partial \zeta^{2}} .
\end{aligned}
$$

The series solutions are obtained by using the numerical values $d=0.8, m=160, b=3, n=120, a=0.5$, and $c=1$.

By comparing the coefficients of powers $p$, we get

$$
\begin{aligned}
p^{0}: \mu_{0}(\zeta, \Psi) & =160 e^{-\zeta^{2}}, \\
p^{0}: \rho_{0}(\zeta, \Psi) & =120 e^{-\zeta^{2}}, \\
p^{1}: \mu_{1}(\zeta, \Psi) & =N^{-}\left[\frac{u^{\gamma}}{s^{\gamma}} N\left[a \frac{\partial^{2} \mu_{0}(\zeta, \Psi)}{\partial \zeta^{2}}-\mathscr{H}_{0}+\mathscr{H}_{0}^{\prime}\right]\right], \\
\mu_{1}(\zeta, \Psi) & =4\left\{\left(9600 \zeta^{2}-40\right) e^{-\zeta^{2}}-60\right\} e^{-\zeta^{2}} \frac{\Psi^{\gamma}}{\Gamma(\gamma+1)}, \\
\rho_{1}(\zeta, \Psi) & \left.=8\left\{3\left(60 \zeta^{2}-30\right)+8\right)\right\} e^{-\zeta^{2}} \frac{\Psi^{\gamma}}{\Gamma(\gamma+1)},
\end{aligned}
$$




$$
\begin{aligned}
p^{2}: \mu_{2}(\zeta, \Psi)= & N^{-}\left\{\frac{u^{\gamma}}{s^{\gamma}} N\left[a \frac{\partial^{2} \mu_{1}(\zeta, \Psi)}{\partial \zeta^{2}}-\mathscr{H}_{1}+\mathscr{H}_{1}^{\prime}\right]\right\}, \\
\mu_{2}(\zeta, \Psi)= & 160\left\{120-240-\left(240 \zeta^{2}-1\right) e^{-\zeta^{2}}-2\left[240 \zeta+\zeta^{2}\left(240 \zeta^{2}-1\right)\right] e^{-\zeta^{2}}\right\} \frac{\Psi^{2 \gamma}}{\Gamma(2 \gamma+1)} \\
& +7680\left\{120-4 \zeta\left[15\left(2 \zeta^{2}-1\right)+2\right]\right\} \zeta e^{-2 \zeta^{2}} \\
& -\left\{\left(38400\left(480+160\left(2 \zeta^{2}-1\right)\right)-960\right) e^{-2 \zeta^{2}}+500(307-614.4 \zeta) \zeta e^{-3 \zeta^{2}}\right\} \\
& \cdot \frac{\Psi^{3 \gamma}}{\Gamma(3 \gamma+1)}+\left\{\begin{array}{c}
1280\left(720-2\left(90\left(2 \zeta^{2}-1\right)+8\right)+4 \zeta^{2}\left(90\left(2 \zeta^{2}-1\right)+8\right)\right) \\
\left.+160\left(\left(240 \zeta^{2}-1\right)-480 \zeta^{2}+478.125\right) 120\left(4 \zeta^{2}-1\right)\right)
\end{array}\right\} e^{-\zeta^{2}} \frac{\Psi^{3 \gamma}}{\Gamma(3 \gamma+1)}, \\
& p^{2}: \rho_{2}(\zeta, \Psi) \\
\rho_{2}(\zeta, \Psi)= & \left\{25956+6\left(2 \zeta^{2}-1\right)^{2}+1320\left(1-4 \zeta^{2}\right)+3\left(2 \zeta^{2}-1\right)\right\} e^{-\zeta^{2}} \frac{\Psi^{2 \gamma}}{\Gamma(2 \gamma+1)} .
\end{aligned}
$$

The series form of equation (30) is given by

$$
\begin{aligned}
& \mu(\zeta, \Psi)=\sum_{\ell=0}^{\infty} \mu_{\ell}(\zeta, \Psi)=\mu_{0}(\zeta, \Psi)+\mu_{1}(\zeta, \Psi)+\mu_{2}(\zeta, \Psi)+\mu_{3}(\zeta, \Psi)+\cdots \\
& \rho(\zeta, \Psi)=\sum_{\ell=0}^{\infty} \rho_{\ell}(\zeta, \Psi)=\rho_{0}(\zeta, \Psi)+\rho_{1}(\zeta, \Psi)+\rho_{2}(\zeta, \Psi)+\rho_{3}(\zeta, \Psi)+\cdots
\end{aligned}
$$

The HPTM solution is given as

$$
\begin{aligned}
\mu(\zeta, \Psi)= & 160 e^{-\zeta^{2}}+4\left[\left(9600 \zeta^{2}-40\right) e^{-\zeta^{2}}-60\right] e^{-\zeta^{2}} \frac{\Psi^{\gamma}}{\Gamma(\gamma+1)} \\
& +160\left\{120-240-\left(240 \zeta^{2}-1\right) e^{-\zeta^{2}}-2\left[240 \zeta+\zeta^{2}\left(240 \zeta^{2}-1\right)\right] e^{-\zeta^{2}}\right\} \frac{\Psi^{2 \gamma}}{\Gamma(2 \gamma+1)} \\
& +7680\left\{120-4 \zeta\left[15\left(2 \zeta^{2}-1\right)+2\right]\right\} \zeta e^{-2 \zeta^{2}} \\
& -\left\{\left(38400\left[480+160\left(2 \zeta^{2}-1\right)\right]-960 e^{-2 \zeta^{2}}+500(307-614.4 \zeta) \zeta e^{-3 \zeta^{2}}\right)\right\} \\
& \cdot \frac{\Psi^{3 \gamma}}{\Gamma(3 \gamma+1)}+\left\{1280\left(720-2\left(90\left(2 \zeta^{2}-1\right)+8\right)+4 \zeta^{2}\left(90\left(2 \zeta^{2}-1\right)\right)+8\right)\right) \\
& \left.+160\left[\left(240 \zeta^{2}-1\right)-480 \zeta^{2}+478.125\right]\left(120\left(4 \zeta^{2}-1\right)\right)\right\} e^{-\zeta^{2}} \frac{\Psi^{3 \gamma}}{\Gamma(3 \gamma+1)}+\cdots \\
& +\left\{25956+6\left(2 \zeta^{2}-1\right)^{2}+1320(1-4 \zeta)^{2}+3\left(2 \zeta^{2}-1\right)\right\} e^{-\zeta^{2}} \frac{\Psi^{2 \gamma}}{\Gamma(2 \gamma+1)}+\cdots \\
\rho(\zeta, \Psi)= & 120 e^{-\zeta^{2}}+8\left\{3\left(60 \zeta^{2}-30\right)+8\right\} e^{-\zeta^{2}} \frac{\Psi^{\gamma}}{\Gamma(\gamma+1)} \\
&
\end{aligned}
$$




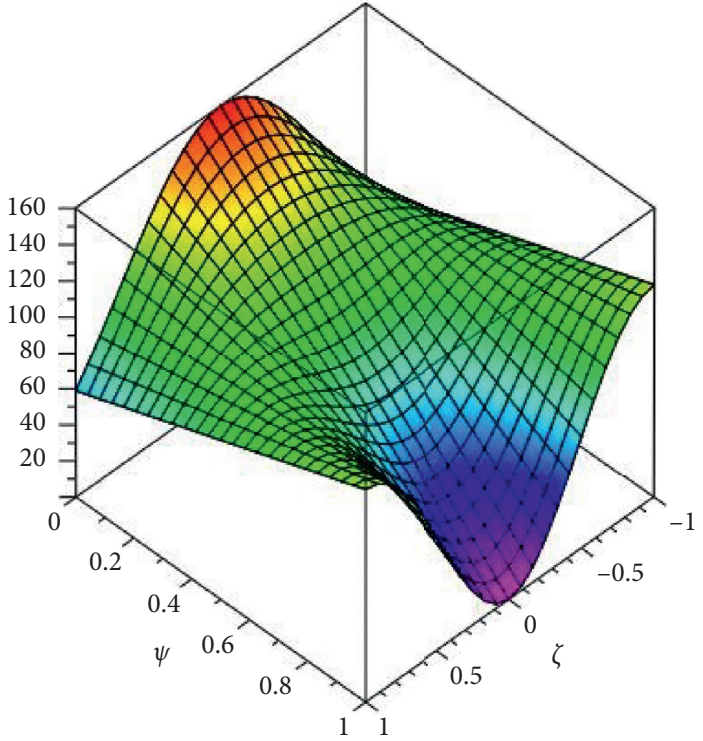

(a)

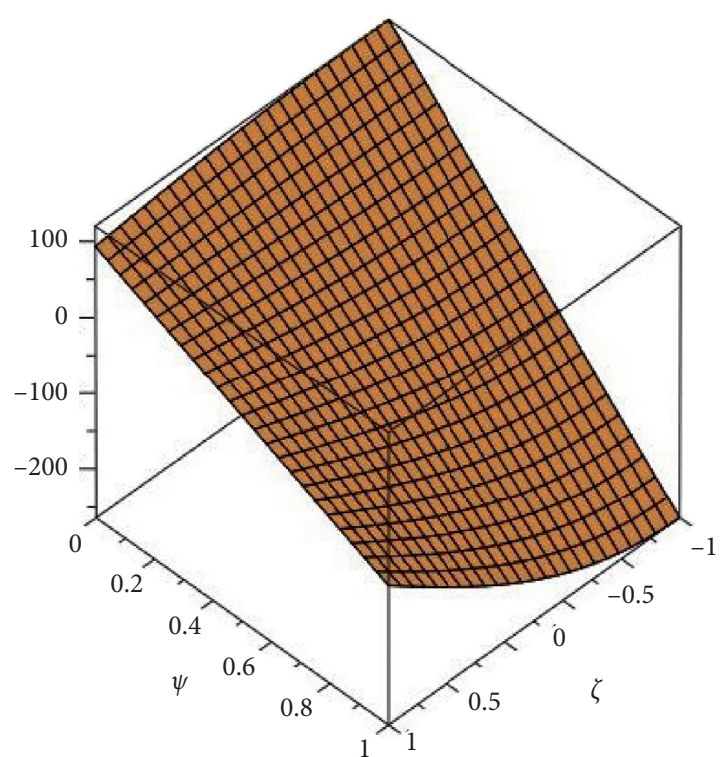

(b)

FIGURE 1: HPTM solutions of $\mu(\zeta, \Psi)$ and $\rho(\zeta, \Psi)$ at $\gamma=1$.

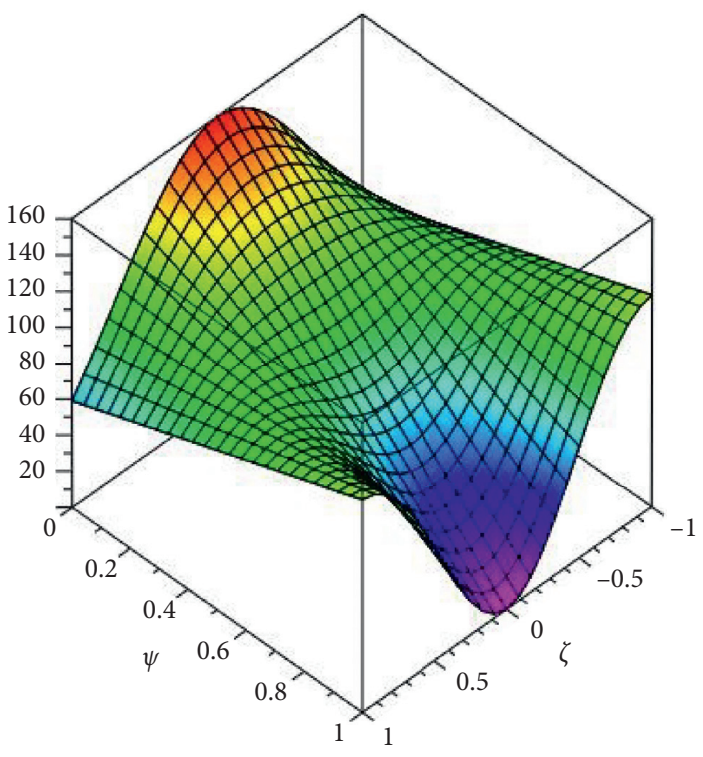

(a)

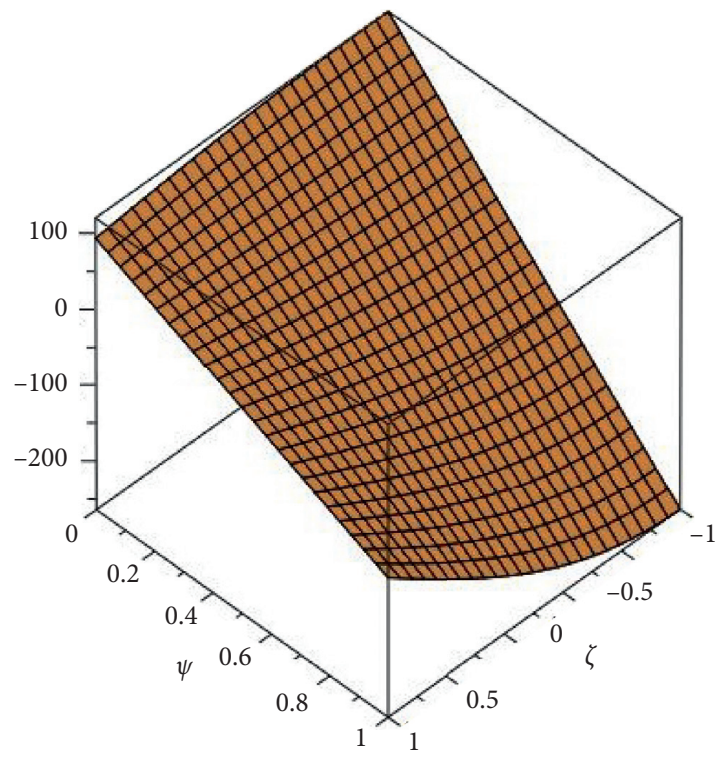

(b)

Figure 2: LADM solutions of $\mu(\zeta, \Psi)$ and $\rho(\zeta, \Psi)$ at $\gamma=1$.

\section{Results and Discussion}

In Figures 1 and 2, the comparative study of HPTM and LADM solutions has been made for variables $\mu(\zeta, \Psi)$ and $\rho(\zeta, \Psi)$ at $\gamma=1$ for Example 1. The graphical representation has shown the close relation between HPTM and LADM solutions. In Figures 3 and 4 , the graphs of $\mu(\zeta, \Psi)$ and $\rho(\zeta, \Psi)$ verses $\zeta$ are plotted for fixed $\Psi=1$ for both fractional and integer orders of Example 2. The graphical representation has confirmed the convergence of fractional-order solutions towards integer-order solutions. In Table 1, the LADM and HPTM solutions of Example 1 are compared at $\zeta=1$ and $\gamma=1$. It is observed that LADM and HPTM solutions are identical and justify the reliability of the proposed techniques. In Figures 5 and 6, the HPTM solutions, and in Figure 7, the HPTM solutions at fractional orders $\gamma=0.5,0.6,0.8$, and 1 , are discussed for variables $\mu(\zeta, \Psi)$ and $\rho(\zeta, \Psi)$. It is investigated that both the methods have a higher degree of accuracy and provide the closedform solution to Example 2. 


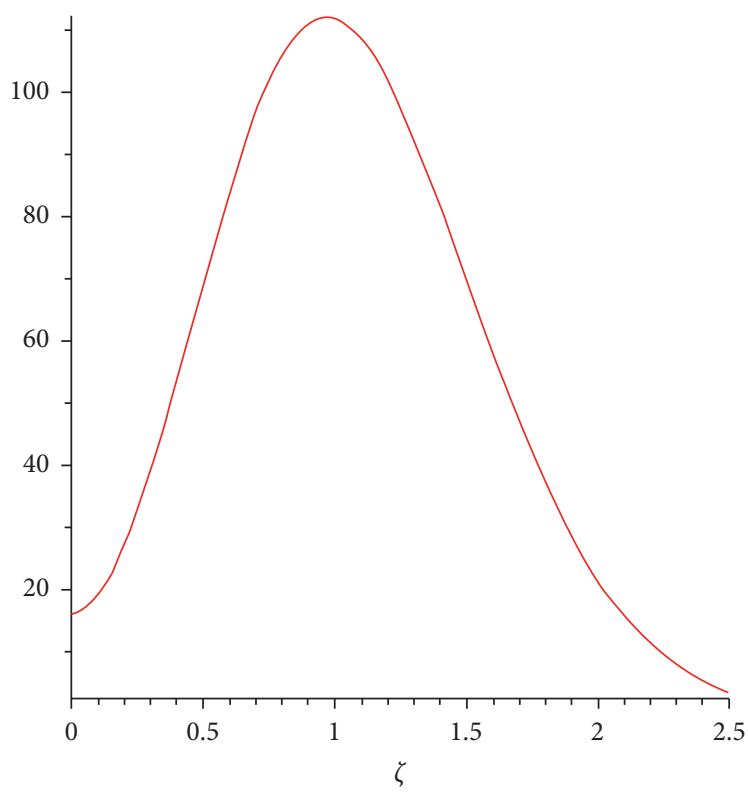

(a)

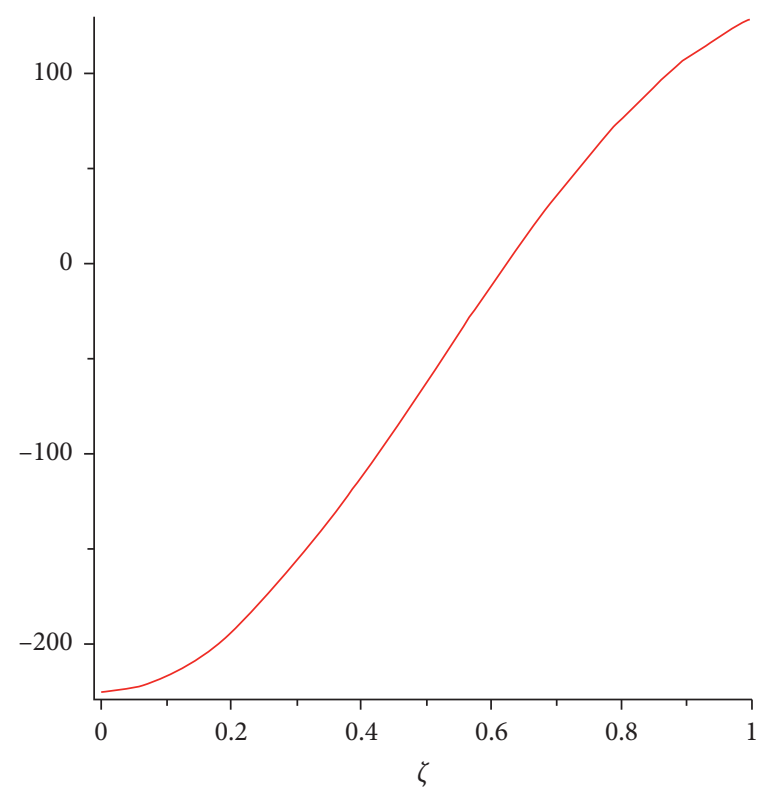

(b)

FIGURE 3: HPTM solutions of $\mu(\zeta, \Psi)$ and $\rho(\zeta, \Psi)$ at $\gamma=1$.

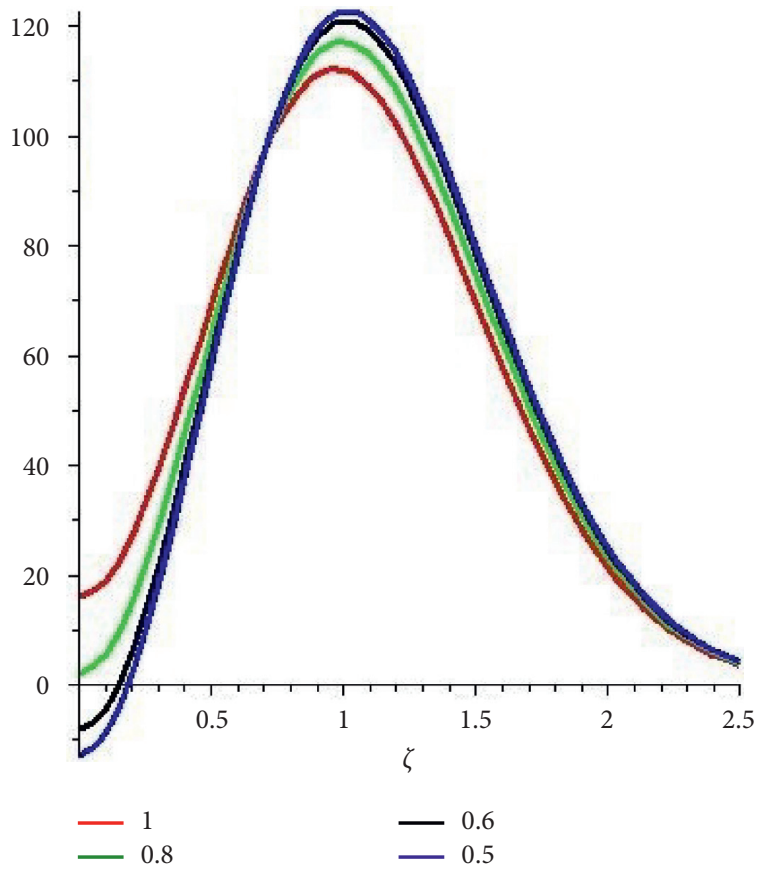

(a)

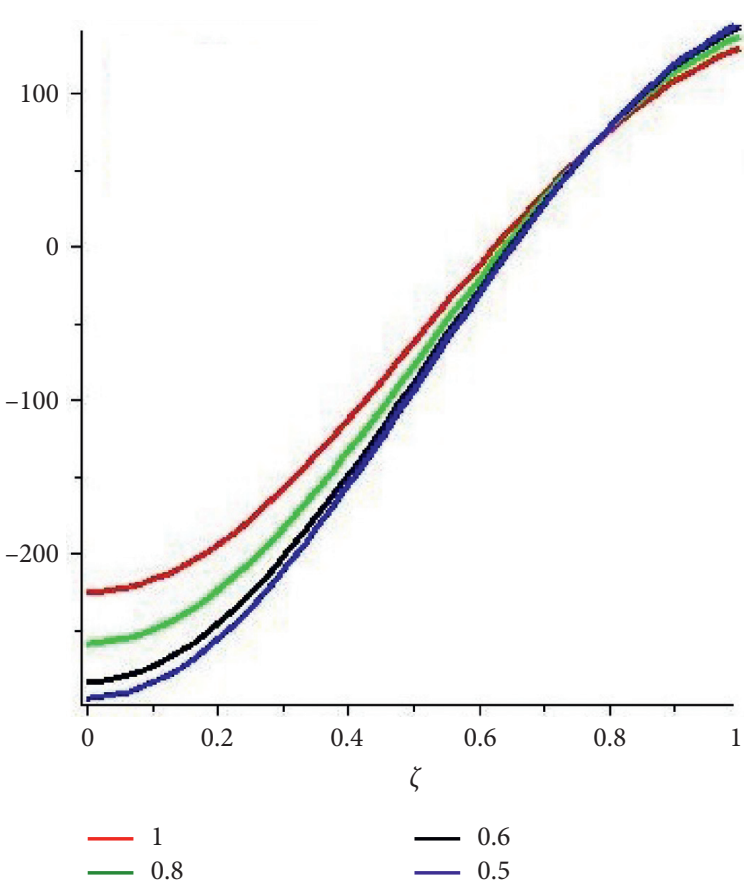

(b)

FIGURE 4: HPTM solutions of $\mu(\zeta, \Psi)$ and $\rho(\zeta, \Psi)$ at different fractional orders $\gamma=0.5,0.6,0.8,1$ and $\Psi=1$.

TABLE 1: Comparison of HDM [36], LADM [37], and HPTM at $\zeta=1$ and $\gamma=1$.

\begin{tabular}{lccccc}
\hline$\Psi$ & HDM & HDM & LADM & LADM & HPTM \\
& $\mu(\zeta, \psi)$ & $\rho(\zeta, \psi)$ & $\mu(\zeta, \psi)$ & $\rho(\zeta, \psi)$ & $\begin{array}{c}\text { HPTM } \\
\rho, \psi)\end{array}$ \\
\hline 0.2 & $2.70 \times 10^{5}$ & $8.0 \times 10^{2}$ & $2.70 \times 10^{5}$ & $8.0 \times 10^{2}$ & $2.70 \times 10^{5}$ \\
0.4 & $3.0 \times 10^{6}$ & $2.0 \times 10^{3}$ & $3.0 \times 10^{6}$ & $2.0 \times 10^{3}$ & $3.0 \times 10^{6}$ \\
0.6 & $5.90 \times 10^{6}$ & $4.0 \times 10^{3}$ & $5.90 \times 10^{6}$ & $4.0 \times 10^{3}$ & $5.90 \times 10^{6}$ \\
0.8 & $8.50 \times 10^{6}$ & $7.0 \times 10^{3}$ & $8.50 \times 10^{6}$ & $7.0 \times 10^{3}$ & $8.50 \times 10^{6}$ \\
1.00 & $1.50 \times 10^{7}$ & $1.20 \times 10^{4}$ & $1.50 \times 10^{7}$ & $1.20 \times 10^{4}$ & $1.50 \times 10^{7}$ \\
\hline
\end{tabular}




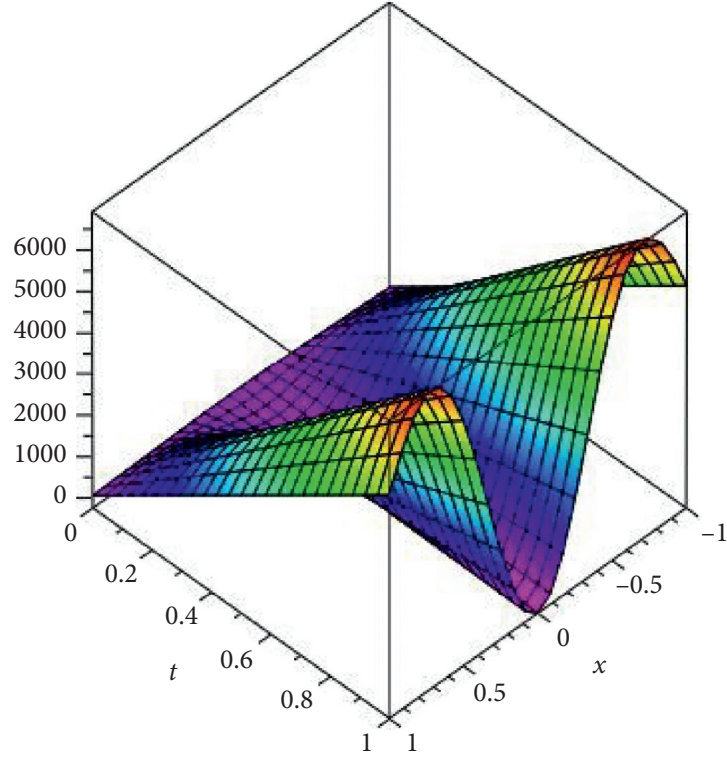

(a)

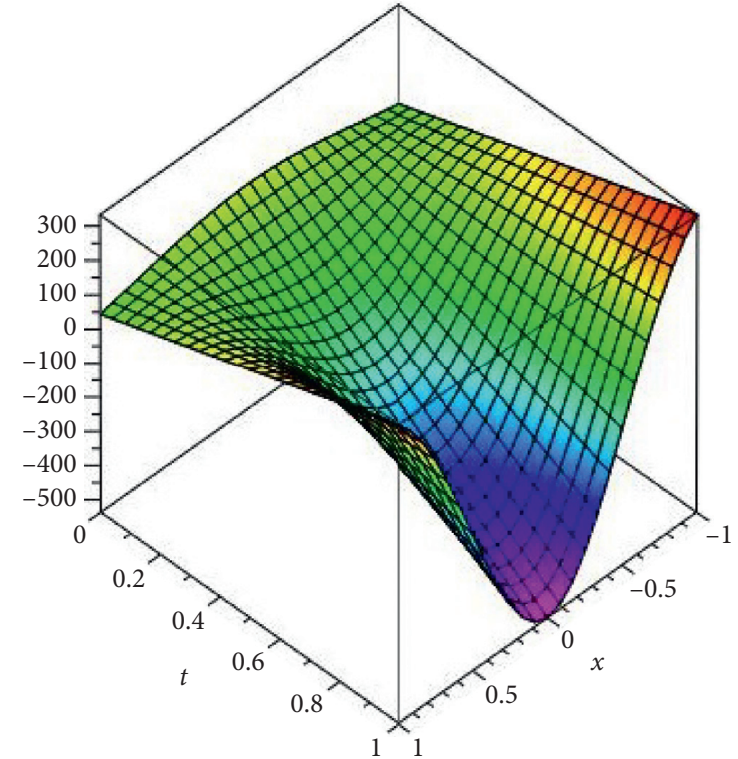

(b)

FiguRE 5: HPTM solutions of $\mu(\zeta, \Psi)$ and $\rho(\zeta, \Psi)$ at $\gamma=1$.

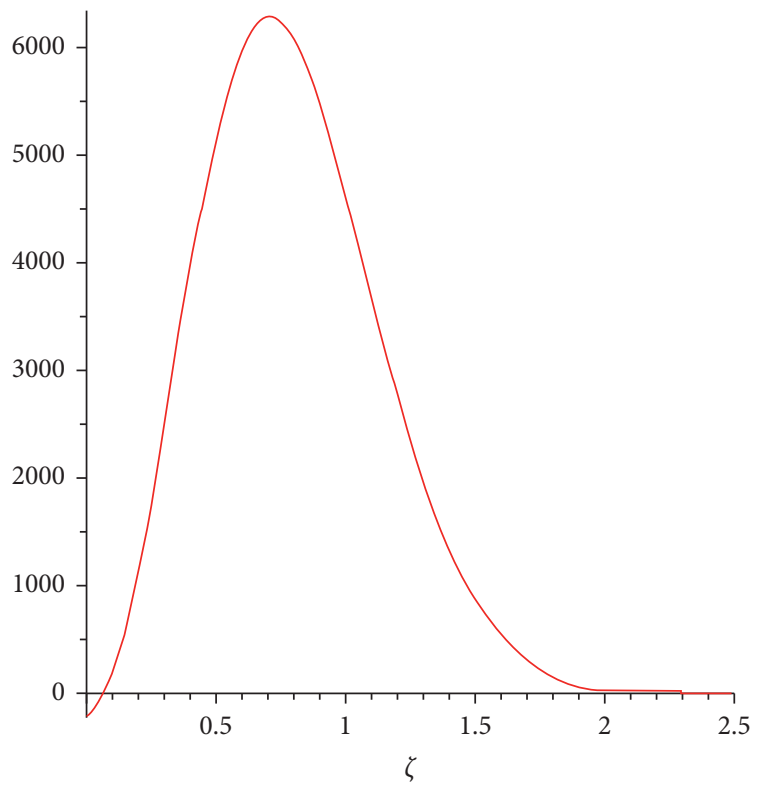

(a)

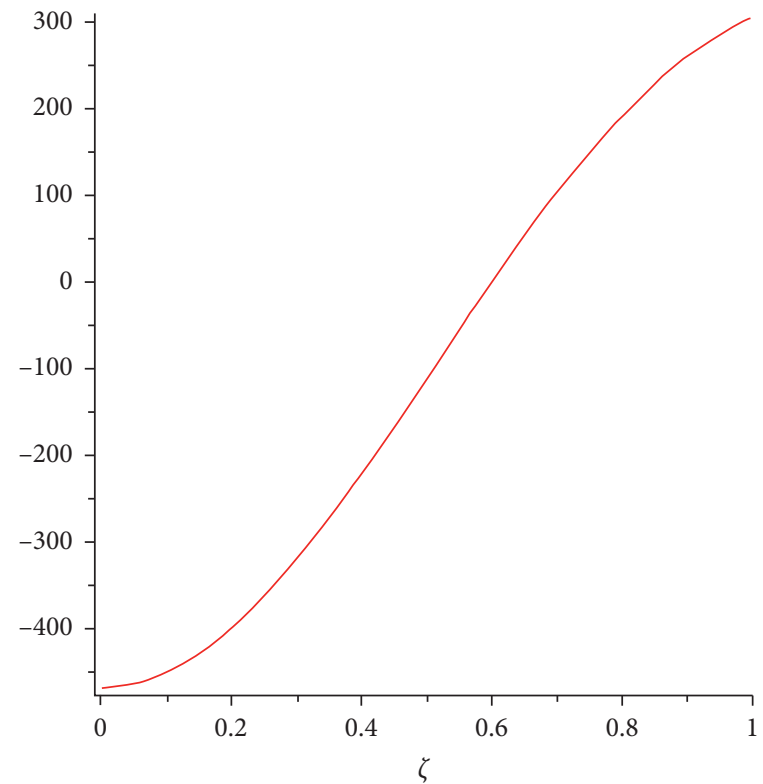

(b)

FIGURE 6: HPTM solutions of $\mu(\zeta, \Psi)$ and $\rho(\zeta, \Psi)$ at $\gamma=1$. 


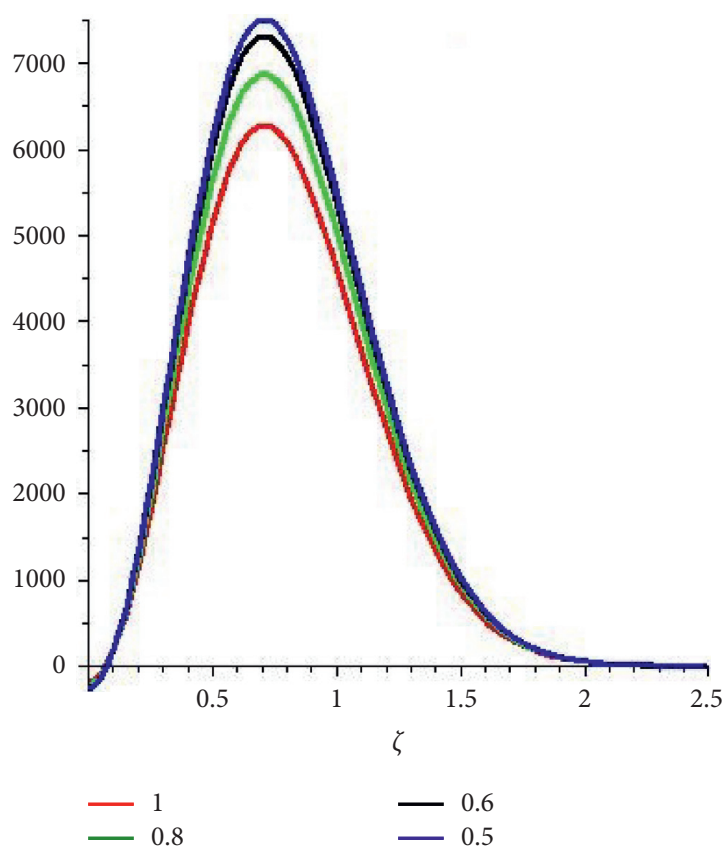

(a)

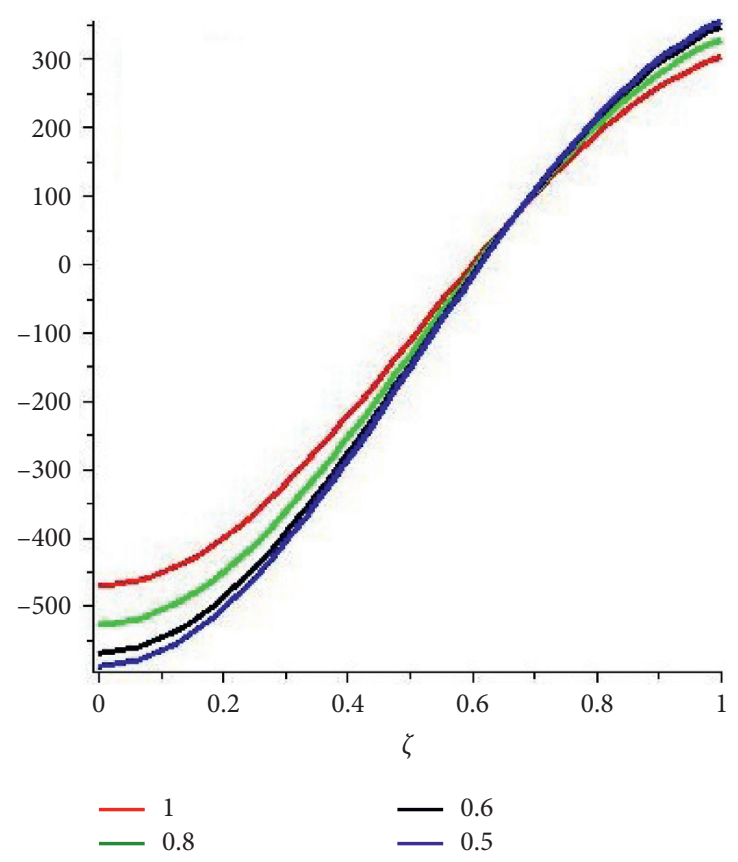

(b)

Figure 7: HPTM solutions of $\mu(\zeta, \Psi)$ and $\rho(\zeta, \Psi)$ at different fractional orders $\gamma=0.5,0.6,0.8,1$ and $\Psi=1$.

\section{Conclusion}

In the current article, an effective technique which is known as the Laplace homotopy transform method is implemented to solve the systems of Keller-Segal equations for both fractional and integer orders of the derivatives within the Caputo operator. Two numerical examples of fractional Keller-Segal equations are presented to verify the reliability of the suggested method. The graphical and tabular representation has confirmed that the derived results are in close agreement with the solution of the Laplace Adomian decomposition method. Moreover, the current technique needs very small calculations and has a higher degree of accuracy for the targeted problems. In conclusion, the present technique is found to be an accurate and effective analytical technique to solve high nonlinear fractional systems of partial differential equations.

\section{Data Availability}

The numerical data used to support the findings of this study are included within the article.

\section{Conflicts of Interest}

The authors declare that there are no conflicts of interest regarding the publication of this article.

\section{Acknowledgments}

The authors extend their appreciation to the Deanship of Scientific Research at King Khalid University, Saudi Arabia, for funding this work through the research groups program under the grant number (R.G.P2./99/41).

\section{References}

[1] K. S. Miller and B. Ross, An Introduction to the Fractional Calculus and Fractional Differential Equations, Wiley, Hoboken, NJ, USA, 1993.

[2] R. Hilfer, Applications of Fractional Calculus in Physics, World Science Publishing, River Edge, NJ, USA, 2000.

[3] I. Podlubny, "Fractional differential equations," in An Introduction to Fractional Derivatives, Fractional Differential Equations, to Methods of Their Solution and Some of Their Applications, Elsevier, Amsterdam, Netherlands, 1998.

[4] L. Debnath, "Recent applications of fractional calculus to science and engineering," International Journal of Mathematics and Mathematical Sciences, vol. 2003, no. 54, pp. 3413-3442, 2003.

[5] M. Ortigueira, "An introduction to the fractional continuoustime linear systems: the 21st century systems," IEEE Circuits and Systems Magazine, vol. 8, no. 3, pp. 19-26, 2008.

[6] T. Kaczorek, "Positive linear systems consisting of $\$ \mathrm{n} \$$ subsystems with different fractional orders," IEEE Transactions on Circuits and Systems I: Regular Papers, vol. 58, no. 6, pp. 1203-1210, 2011.

[7] R. Shah, H. Khan, S. Mustafa, P. Kumam, and M. Arif, "Analytical solutions of fractional-order diffusion equations by natural transform decomposition method," Entropy, vol. 21, no. 6, p. 557, 2019.

[8] R. Jan and Y. Xiao, "Effect of partial immunity on transmission dynamics of dengue disease with optimal control," Mathematical Methods in the Applied Sciences, vol. 42, no. 6, pp. 1967-1983, 2019.

[9] R. Jan and Y. Xiao, "Effect of pulse vaccination on dynamics of dengue with periodic transmission functions," Advances in Difference Equations, vol. 1, p. 368, 2019.

[10] A. A. Kilbas, H. M. Srivastava, and J. J. Trujillo, Theory and applications of fractional differential equations, Vol. 204, Elsevier, Amsterdam, Netherlands, 2006. 
[11] V. Lakshmikantham and A. S. Vatsala, "Basic theory of fractional differential equations," Nonlinear Analysis: Theory, Methods \& Applications, vol. 69, no. 8, pp. 2677-2682, 2008.

[12] K. B. Oldham, "Fractional differential equations in electrochemistry," Advances in Engineering Software, vol. 41, no. 1, pp. 9-12, 2010.

[13] B. Lu, "Bäcklund transformation of fractional Riccati equation and its applications to nonlinear fractional partial differential equations," Physics Letters A, vol. 376, no. 28-29, pp. 20452048, 2012.

[14] Z. Odibat and S. Momani, "The variational iteration method: an efficient scheme for handling fractional partial differential equations in fluid mechanics," Computers \& Mathematics with Applications, vol. 58, no. 11-12, pp. 2199-2208, 2009.

[15] H. Khan, R. Shah, P. Kumam, and M. Arif, "Analytical solutions of fractional-order heat and wave equations by the natural transform decomposition method," Entropy, vol. 21, no. 6, p. 597, 2019.

[16] S. Mahmood, R. Shah, H. khan, and M. Arif, "Laplace adomian decomposition method for multi dimensional time fractional model of Navier-Stokes equation," Symmetry, vol. 11, no. 2, p. 149, 2019.

[17] R. Shah, H. Khan, D. Baleanu, P. Kumam, and M. Arif, "A novel method for the analytical solution of fractional ZakharovKuznetsov equations," Advances in Difference Equations, vol. 2019, no. 1, pp. 1-14, 2019.

[18] H. M. Srivastava, R. Shah, H. Khan, and M. Arif, "Some analytical and numerical investigation of a family of fractional-order Helmholtz equations in two space dimensions," Mathematical Methods in the Applied Sciences, vol. 43, no. 1, pp. 199-212, 2020.

[19] H. Khan, R. Shah, M. Arif, and S. Bushnaq, "The Chebyshev wavelet method (CWM) for the numerical solution of fractional HIV infection of CD4 + T cells model," International Journal of Applied and Computational Mathematics, vol. 6, no. 2, pp. 1-17, 2020.

[20] Q. M. Al-Mdallal, M. I. Syam, and M. N. Anwar, “A collocation-shooting method for solving fractional boundary value problems," Communications in Nonlinear Science and $\mathrm{Nu}$ merical Simulation, vol. 15, no. 12, pp. 3814-3822, 2010.

[21] M. A. Hajji, Q. M. Al-Mdallal, and F. M. Allan, "An efficient algorithm for solving higher-order fractional Sturm-Liouville eigenvalue problems," Journal of Computational Physics, vol. 272, pp. 550-558, 2014.

[22] Q. M. Al-Mdallal and M. A. Hajji, “A convergent algorithm for solving higher-order nonlinear fractional boundary value problems," Fractional Calculus and Applied Analysis, vol. 18, no. 6, p. 1423, 2015.

[23] Q. M. Al-Mdallal, "On fractional-Legendre spectral Galerkin method for fractional Sturm-Liouville problems," Chaos, Solitons \& Fractals, vol. 116, pp. 261-267, 2018.

[24] R. Shah, H. Khan, D. Baleanu, P. Kumam, and M. Arif, “A semi-analytical method to solve family of Kuramoto-Sivashinsky equations," Journal of Taibah University for Science, vol. 14, no. 1, pp. 402-411, 2020.

[25] M. Madani, M. Fathizadeh, Y. Khan, and A. Yildirim, "On the coupling of the homotopy perturbation method and Laplace transformation," Mathematical and Computer Modelling, vol. 53, no. 9-10, pp. 1937-1945, 2011.

[26] Y. Khan and Q. Wu, "Homotopy perturbation transform method for nonlinear equations using He's polynomials," Computers \& Mathematics with Applications, vol. 61, no. 8, pp. 1963-1967, 2011.
[27] S. J. Johnston, H. Jafari, S. P. Moshokoa, V. M. Ariyan, and D. Baleanu, "Laplace homotopy perturbation method for Burgers equation with space- and time-fractional order," Open Physics, vol. 14, no. 1, pp. 247-252, 2016.

[28] V. F. Morales-Delgado, J. F. Gómez-Aguilar, H. YépezMartínez, D. Baleanu, R. F. Escobar-Jimenez, and V. H. Olivares-Peregrino, "Laplace homotopy analysis method for solving linear partial differential equations using a fractional derivative with and without kernel singular," Advances in Difference Equations, vol. 2016, no. 1, pp. 1-17, 2016.

[29] Y. Li, B. T. Nohara, and S. Liao, "Series solutions of coupled Van der Pol equation by means of homotopy analysis method," Journal of Mathematical Physics, vol. 51, no. 6, Article ID 063517, 2010.

[30] Y. Keskin and G. Oturanc, "Reduced differential transform method for partial differential equations," International Journal of Nonlinear Sciences and Numerical Simulation, vol. 10, no. 6, pp. 741-750, 2009.

[31] P. K. Gupta, "Approximate analytical solutions of fractional Benney-Lin equation by reduced differential transform method and the homotopy perturbation method," Computers \& Mathematics with Applications, vol. 61, no. 9, pp. 28292842, 2011.

[32] K. H. Huebner, D. L. Dewhirst, D. E. Smith, and T. G. Byrom, The Finite Element Method for Engineers, John Wiley \& Sons, Hoboken, NJ, USA, 2001.

[33] G. D. Smith, G. D. Smith, and G. D. S. Smith, Numerical Solution of Partial Differential Equations: Finite Difference Methods, Oxford University Press, Oxford, UK, 1985.

[34] R. Shah, H. Khan, and D. Baleanu, "Fractional whithambroer-kaup equations within modified analytical approaches," Axioms, vol. 8, no. 4, p. 125, 2019.

[35] E. F. Keller and L. A. Segel, "Initiation of slime mold aggregation viewed as an instability," Journal of Theoretical Biology, vol. 26, no. 3, pp. 399-415, 1970.

[36] J. Lankeit and M. Winkler, A Generalized Solution Concept for the Keller-Segel System with Logarithmic Sensitivity: Global Solvability for Large Nonradial Data, https://arxiv.org/abs/ 1701.07391, 2017.

[37] F. Haq, K. Shah, Q. M. Al-Mdallal, and F. Jarad, “Application of a hybrid method for systems of fractional order partial differential equations arising in the model of the one-dimensional Keller-Segel equation," The European Physical Journal Plus, vol. 134, no. 9, p. 461, 2019.

[38] N. Bournaveas and V. Calvez, "The one-dimensional KellerSegel model with fractional diffusion of cells," Nonlinearity, vol. 23, no. 4, pp. 923-935, 2010.

[39] M. Zayernouri and A. Matzavinos, "Fractional AdamsBashforth/Moulton methods: an application to the fractional Keller-Segel chemotaxis system," Journal of Computational Physics, vol. 317, pp. 1-14, 2016.

[40] V. F. Morales-Delgado, J. F. Gómez-Aguilar, S. Kumar, and M. A. Taneco-Hernández, "Analytical solutions of the KellerSegel chemotaxis model involving fractional operators without singular kernel," The European Physical Journal Plus, vol. 133, no. 5, p. 200, 2018.

[41] M. Winkler, "The two-dimensional Keller-Segel system with singular sensitivity and signal absorption: global large-data solutions and their relaxation properties," Mathematical Models and Methods in Applied Sciences, vol. 26, no. 5, pp. 987-1024, 2016.

[42] Y. Dolak and C. Schmeiser, "The keller--segel model with logistic sensitivity function and small diffusivity," SIAM 
Journal on Applied Mathematics, vol. 66, no. 1, pp. 286-308, 2005.

[43] S. Kumar, A. Kumar, and I. K. Argyros, "A new analysis for the Keller-Segel model of fractional order," Numerical Algorithms, vol. 75, no. 1, pp. 213-228, 2017.

[44] A. Ghorbani and J. Saberi-Nadjafi, "He's homotopy perturbation method for calculating adomian polynomials," International Journal of Nonlinear Sciences and Numerical Simulation, vol. 8, no. 2, pp. 229-232, 2007.

[45] A. Ghorbani, "Beyond Adomian polynomials: he polynomials," Chaos, Solitons \& Fractals, vol. 39, no. 3, pp. 1486-1492, 2009.

[46] H. Khan, R. Shah, D. Baleanu, P. Kumam, and M. Arif, "Analytical solution of fractional-order hyperbolic telegraph equation, using natural transform decomposition method," Electronics, vol. 8, no. 9, p. 1015, 2019.

[47] S. Maitama, "A hybrid natural transform homotopy perturbation method for solving fractional partial differential equations," International Journal of Differential Equations, vol. 2016, Article ID 9207869, 7 pages, 2016.

[48] S. Maitama, "Local fractional natural homotopy perturbation method for solving partial differential equations with local fractional derivative," Progress in Fractional Differentiation and Applications, vol. 4, no. 3, pp. 219-228, 2018.

[49] A. Khalouta and A. Kadem, "Comparison of new iterative method and natural homotopy perturbation method for solving nonlinear time-fractional wave-like equations with variable coefficients," Nonlinear Dynamics and Systems Theory, vol. 19, pp. 160-169, 2019.

[50] Z. H. Khan and W. A. Khan, "N-transform-properties and applications," NUST Journal of Engineering Sciences, vol. 1, no. 1, pp. 127-133, 2008.

[51] K. Shah, H. Khalil, and R. A. Khan, "Analytical solutions of fractional order diffusion equations by natural transform method," Iranian Journal of Science and Technology, Transactions A: Science, vol. 42, no. 3, pp. 1479-1490, 2018. 